Bull. Chem. Soc. Ethiop. 2018, 32(2), 259-270.

ISSN 1011-3924

(c) 2018 Chemical Society of Ethiopia and The Authors

Printed in Ethiopia

DOI: https://dx.doi.org/10.4314/bcse.v32i2.7

\title{
SYNTHESIS AND CYTOTOXICITY OF NOVEL THIOPHENE, PYRAN AND PYRIDINE DERIVATIVES
}

\author{
Wagnat W. Wardakhan*, Eman M. Samir and Elham E. El-Arab \\ Department of Organic Chemistry, National Organization for Drug Control and Research \\ (NODCAR), Giza 12311, Egypt
}

(Received January 22, 2018; Revised March 5, 2018; Accepted March 9, 2018)

\begin{abstract}
The reaction of 2-amino-3-cyano-tetrahydrobenzothiophene 3 with ethyl acetoacetate gave the amide derivatives $\mathbf{5}$. The reactivity of $\mathbf{5}$ toward a variety of chemical reagents was studied to give pyrans, pyridines, thiophenes and the thiazoles and their fused derivatives. The structures of the newly synthesized products were confirmed on the basis of their respective analytical and spectral data. The antitumor evaluations of the synthesized compounds against the three cancer cell lines MCF-7, NCI-H460 and SF-268 showed that compounds 9a, 9c, 12a and 14 were of the highest potencies against the three cancer cell lines among the tested compounds.
\end{abstract}

KEY WORDS: Tetrahydrobenz[b]thiophene, Pyran, Pyridine, Thiophene, Thiazole, Cytotoxicity

\section{INTRODUCTION}

Aromatic thiophenes play on part in animal metabolism; for examples, Biotin, one of the vitamins (Vitamin H) (Figure 1), is a tetrahydrothiophene, however aromatic thiophenes do occur in some plants, in association with polyacetylenes with which they are biogenetically linked and Banminth (pyrantel), available anthelmintic used in animal husbandry, is one of the thiophene compounds in chemotherapy. Thiophenes with a wide spectrum of biological activities are known, several of these derivatives possess potent analgesic $[1,2]$, anticonvulsant, anti-inflammatory and antibacterial [3-6], antipyretics [7], antitumor [8,9], antiparasitic [10], antimicrobial [11], antihistaminic (H1) [12], antianexiety test in mice [13], antiarrhythmic [14] and serotonin antagonist [15]. In previous work we have found that certain substituted thiophenes and their heterocyclic derivatives show antitumor activities [16-18]. In the present work we were aiming the synthesis of new heterocyclic derivatives derived from the 2-amino-3cyano-4,5,6,7-tetrahydrobenzo[b]thiophene followed by studying the cytotoxicity of the newly synthesized compounds.

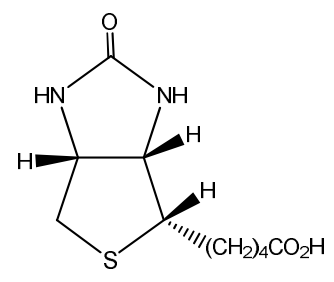

(+)-Biotin (Vitamin H)

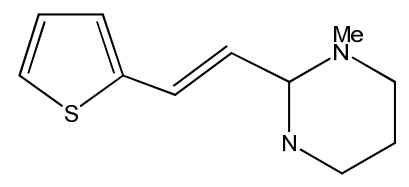

Banminth

Figure 1. Chemical structures of (+)-Biotin and Banminth.

*Corresponding author. E-mail: wagnatwahba@yahoo.com

This work is licensed under the Creative Commons Attribution 4.0 International License 


\section{Chemistry}

\section{RESULTS AND DISCUSSION}

In the present work we synthesised a series of heterocyclic rings incorporated tetrahydrobenzo $[b]$ thiophene moiety through the use of 2-amino-3-cyano-4,5,6,7-tetrahydrobenzo $[b]$ thiophene (3). Compound $\mathbf{3}$ was synthesized via the reaction of cyclohexanone (1) with malononitrile (2) and elemental sulfur [19, 20]. Compound 3 reacted with ethyl acetoacetate (4) in dimethylformamide under reflux to give the amide derivative (5). The multi-component reaction of compound $\mathbf{5}$ and any of the aromatic aldehydes namely benzaldehyde (6a), 4chlorobenzaldehyde (6b) or 4-methoxybenzaldehyde (6c) in 1,4-dioxane containing triethylamine with malononitrile gave the pyran derivatives 7a-c, respectively (Scheme 1). The analytical and spectral data of compounds $7 \mathbf{a}-\mathbf{c}$ were the basis of their structural elucidation. Thus, the ${ }^{1} \mathrm{H}$ NMR spectrum of $7 \mathbf{a}$ (as an example) revealed beside the expected signals a singlet at $\delta 3.19 \mathrm{ppm}$ corresponding to the $\mathrm{CH}_{3}$ group, a singlet at $\delta 6.01 \mathrm{ppm}$ corresponding to the pyran $\mathrm{H}-4$ a multiplet at $\delta 7.28-7.39 \mathrm{ppm}$ equivalent to the $\mathrm{C}_{6} \mathrm{H}_{5}$ group and a singlet at $\delta 8.63$ ppm, $\mathrm{D}_{2} \mathrm{O}$ exchangeable, for the $\mathrm{NH}$ group.
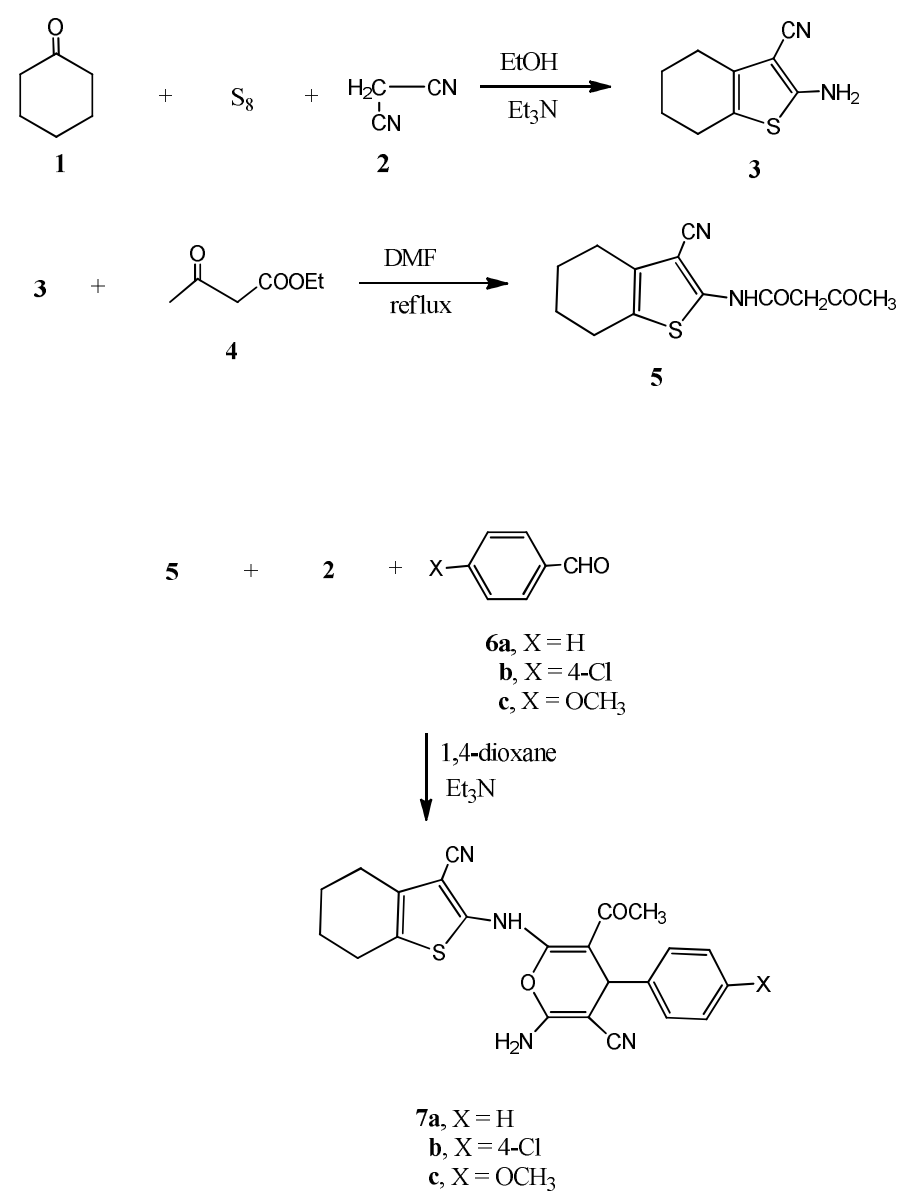

Scheme 1. Synthesis of compounds 5, 6a-c and 7a-c.

Bull. Chem. Soc. Ethiop. 2018, 32(2) 


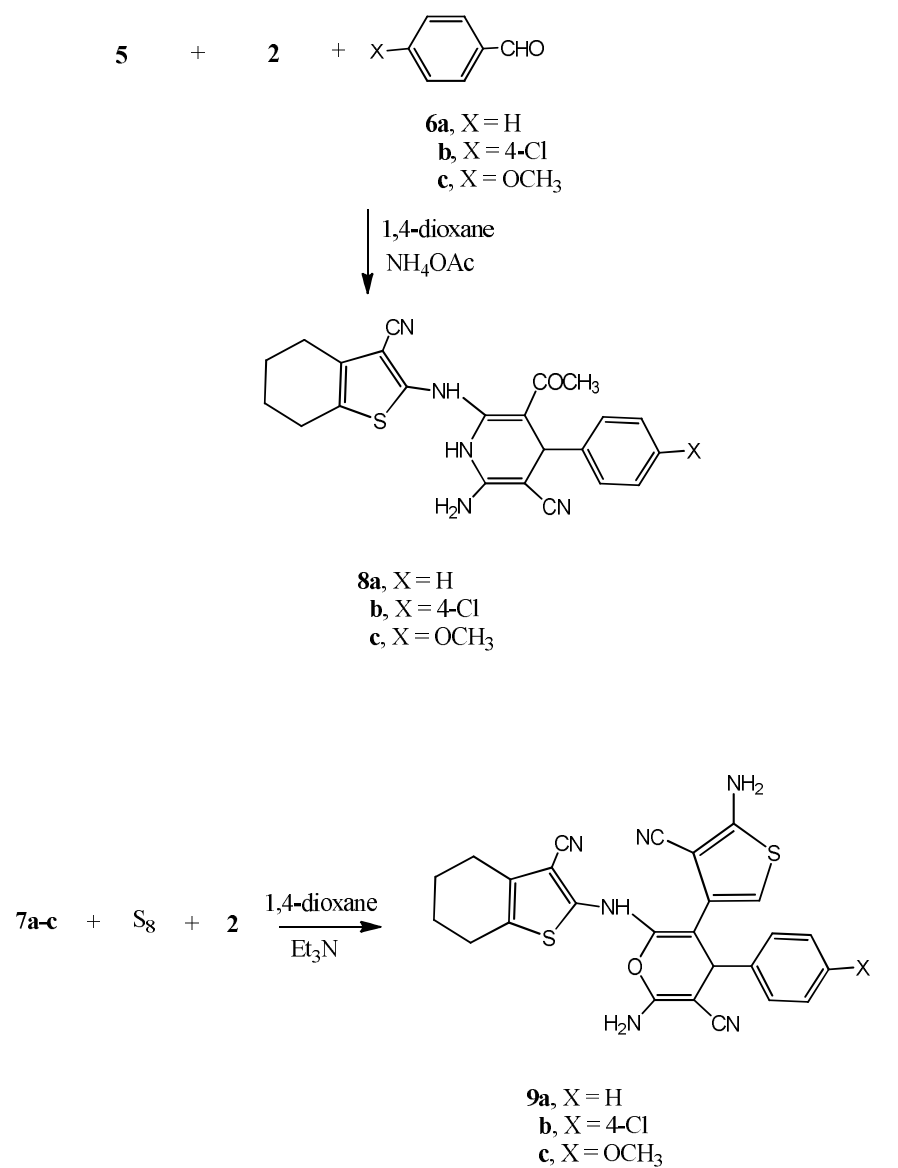

Scheme 2. Synthesis of compounds 8a-c and 9a-c.

Scheme 2. Synthesis of compounds 8a-c and 9a-c.

In addition the ${ }^{13} \mathrm{C}$ NMR spectrum showed, beside the expected signals, $22.8\left(\mathrm{CH}_{3}\right), 88.3$ (pyran C-4), 116.2, $118.3(2 \mathrm{CN}), 120.3,121.3,121.8,122.4,126.0,128.4,124.2,130.8,133.0$, 134.2, 137.3, 139.2 (benzene, pyran, thiophene C), 165.8 (CO). Moreover, the multi-component reaction of compound 5 with any of the aromatic aldehydes namely benzaldehyde (6a), 4chlorobenzaldehyde (6b) or 4-methoxybenzaldehyde (6c) in 1,4-dioxane containing ammonium acetate gave the pyridine derivatives 8a-c. Compounds 7a-c each with the acetyl group at the pyran ring found to be capable for the Gewald's thiophene synthesis. Thus, any of compounds 7a-c reacted with malononitrile and elemental sulfur to give the thiophene derivatives 9a-c, respectively. The analytical and spectral data of 9a-c were consistent with their respective structures as depicted in Scheme 2. Thus, the ${ }^{1} \mathrm{H}$ NMR spectrum of 9a revealed, beside the expected signals, the presence of two singlets at $\delta 4.38,4.70 \mathrm{ppm}\left(\mathrm{D}_{2} \mathrm{O}\right.$ exchangeable $)$ equivalent to the two $\mathrm{NH}_{2}$ groups, a singlet at $\delta 5.96 \mathrm{ppm}$ corresponding to the thiophene $\mathrm{H}-5$, a singlet at $\delta 6.04 \mathrm{ppm}$ for the pyran $\mathrm{H}-4$ and a singlet at $\delta 8.39 \mathrm{ppm}\left(\mathrm{D}_{2} \mathrm{O}\right.$ exchangeable) equivalent for the $\mathrm{NH}$ group. The ${ }^{13} \mathrm{C}$ NMR spectrum showed $\delta 88.6$ (pyran C-4), 115.7, 116.0, 
$116.3(3 \mathrm{CN}), 119.5,120.8,121.3,122.9,123.3,126.9,127.5,130.8,132.6,133.5,137.7,138.5$, 139.7, 140.6, 142.9, 145.2 (benzene, pyran, two thiophene C).

Similarly, the pyridine derivatives 8a-c reacted with malononitrile and elemental sulfur to give the thiophene derivatives $\mathbf{1 0 a}-\mathbf{c}$, respectively. The reaction of compound $\mathbf{5}$ with either of malononitrile (2) or ethyl cyanoacetate (11) and elemental sulfur in 1,4-dioxane containing a catalytic amount of triethylamine gave the thiophene derivatives 12a and 12b, respectively. Analytical and spectral data were the basis of their respective structure elucidation. Finally, the reaction of compound 5 with thioglycollic acid (13) gave the thiazole derivative $\mathbf{1 4}$ (Scheme 3 ).
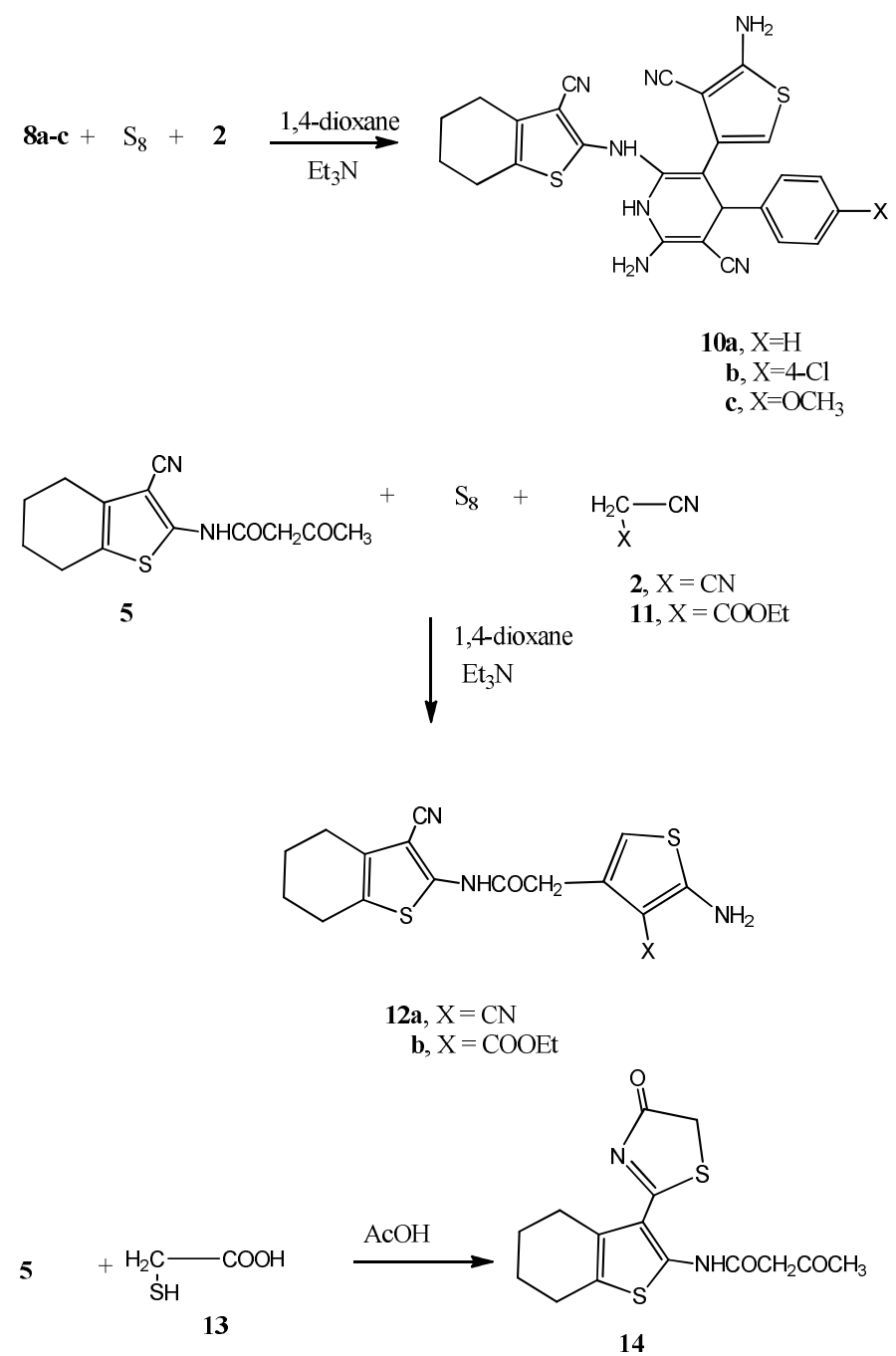

Scheme 3. Synthesis of compounds 10a-c, 12a,b and 14.

Bull. Chem. Soc. Ethiop. 2018, 32(2) 
Antitumor and normal cell line activity tests

Chemicals. Fetal bovine serum (FBS) and L-glutamine were from Gibco Invitrogen Co. (Scotland, UK). RPMI-1640 medium was from Cambrex (New Jersey, USA). Dimethyl sulfoxide (DMSO), doxorubicin, penicillin, streptomycin and sulforhodamine B (SRB) were from Sigma Chemical Co. (Saint Louis, USA).

Cell cultures. Three human tumor cell lines, MCF-7 (breast adenocarcinoma), NCI-H460 (nonsmall cell lung cancer), and SF-268 (CNS cancer) were used. MCF-7 was obtained from the European Collection of Cell Cultures (ECACC, Salisbury, UK), NCI-H460, SF-268 and normal fibroblast cells (WI 38) were kindly provided by the National Cancer Institute (NCI, Cairo, Egypt). They grow as monolayer and routinely maintained in RPMI-1640 medium supplemented with 5\% heat inactivated FBS, $2 \mu \mathrm{M}$ glutamine and antibiotics (penicillin 100 $\mathrm{U} / \mathrm{mL}$, streptomycin $100 \mu \mathrm{g} / \mathrm{mL}$ ), at $37{ }^{\circ} \mathrm{C}$ in a humidified atmosphere containing $5 \% \mathrm{CO}_{2}$. Exponentially growing cells were obtained by plating $1.5 \times 105$ cells $/ \mathrm{mL}$ for MCF-7 and SF-268 and $0.75 \times 104$ cells $/ \mathrm{mL}$ for NCI-H460, followed by $24 \mathrm{~h}$ of incubation. The effect of the vehicle solvent (DMSO) on the growth of these cell lines was evaluated in all the experiments by exposing untreated control cells to the maximum concentration $(0.5 \%)$ of DMSO used in each assay.

Tumor cell growth assay. The effects of the newly synthesized products on the in vitro growth of human tumor cell lines were evaluated according to the procedure adopted by the National Cancer Institute (NCI, USA) in the 'In vitro Anticancer Drug Discovery Screen' that uses the protein-binding dye sulforhodamine $\mathrm{B}$ to assess cell growth. Briefly, exponentially, cells growing in 96-well plates were then exposed for $48 \mathrm{~h}$ to five serial concentrations of each compound, starting from a maximum concentration of $150 \mu \mathrm{M}$. Following this exposure period adherent cells were fixed, washed, and stained. The bound stain was solubilized and the absorbance was measured at $492 \mathrm{~nm}$ in a plate reader (Bio-Tek Instruments Inc., Power wave XS, Wincoski, USA). For each test compound and cell line, a dose-response curve was obtained and the growth inhibition of $50 \%\left(\mathrm{GI}_{50}\right)$, corresponding to the concentration of the compounds that inhibited $50 \%$ of the net cell growth was calculated as described elsewhere. Doxorubicin was used as a positive control and tested in the same manner.

Results are given in concentrations that were able to cause $50 \%$ of cell growth inhibition $\left(\mathrm{GI}_{50}\right)$ after a continuous exposure of $48 \mathrm{~h}$ and show means \pm SEM of three-independent experiments performed in duplicate.

\section{Structure activity relationship}

It is clear from Table 1 that the 2-amino-4,5,6,7-tetrahydrobenzo[b]thiophene-3-carbonitrile (3) showed high cytotoxicity against MCF-7 with $\mathrm{GI}_{50} 0.30 \mu \mathrm{mol} \mathrm{L}^{-1}$ but it showed low cytotoxicity against NCI-H460. The reaction of compound $\mathbf{3}$ with ethyl acetoacetate gave the amide derivative 5 which showed moderate cytotoxicity against NCI-H460 and SF-268 with GI $_{50}$ 's 3.1 and $1.96 \mu \mathrm{mol} \mathrm{L}^{-1}$. For the multi-component products $\mathbf{7 a - c}$ it is obvious that compound $7 \mathbf{c}(\mathrm{X}=$ $\mathrm{OCH}_{3}$ ) showed the highest cytotoxicity among the three compounds. On the other hand, the pyridine derivatives 8a-c were of low potency towards the three cancer lines. The reaction of any of compounds 7a-c with elemental sulfur and malononitrile to yield the thiophene derivatives 9a-c where compounds 9a $(\mathrm{X}=\mathrm{H})$ and $9 \mathbf{c}\left(\mathrm{X}=\mathrm{OCH}_{3}\right)$ showed the highest cytotoxicity among the three compounds. On the other hand, compounds 10a-c showed low potencies. Considering the dithiophene derivatives 12a,b, it is clear that compound 12a with $\mathrm{X}$ $=\mathrm{CN}$ showed the higher potency than $\mathbf{1 2 b}, \mathrm{X}=\mathrm{COOEt}$. It is clear that the presence of the $\mathrm{CN}$ group is responsible for its reactivity. Finally, the thiazole derivative 14 showed high potency against the three cancer cell lines. 
Table 1. Effect of newly synthesized compounds on the growth of three human tumor cell lines.

\begin{tabular}{|l|l|l|l|l|}
\hline Compound & MCF-7 & NCI- H460 & SF-268 & WI 38 \\
\hline & $0.30 \pm 0.02$ & $1.80 \pm 0.09$ & $4.20 \pm 1.04$ & $>100$ \\
\hline 3 & $22.3 \pm 0.2$ & $3.1 \pm 0.22$ & $1.96 \pm 0.09$ & $>100$ \\
\hline $\mathbf{5}$ & $22.4 \pm 2.10$ & $10.42 \pm 3.01$ & $8.63 \pm 2.83$ & $>100$ \\
\hline 7a & $23.55 \pm 4.06$ & $34.6 \pm 12.06$ & $45.41 \pm 2.16$ & $>100$ \\
\hline 7b & $1.2 \pm 0.4$ & $0.3 \pm 0.16$ & $2.8 \pm 0.06$ & $>100$ \\
\hline 7c & $26.6 \pm 8.5$ & $29.3 \pm 12.3$ & $18.4 \pm 2.8$ & $68.2 \pm 2.0$ \\
\hline 8a & $22.4 \pm 5.8$ & $26.7 \pm 8.2$ & $31.4 \pm 2.4$ & $>100$ \\
\hline 8b & $20.8 \pm 8.30$ & $22.8 \pm 4.32$ & $22.8 \pm 6.23$ & $>100$ \\
\hline 8c & $0.01 \pm 0.001$ & $0.02 \pm 0.006$ & $0.06 \pm 0.002$ & $>100$ \\
\hline 9a & $24.1 \pm 10.4$ & $30.8 \pm 10.8$ & $26.1 \pm 2.8$ & $25.2 \pm 0.8$ \\
\hline 9b & $0.08 \pm 0.004$ & $0.05 \pm 0.002$ & $0.06 \pm 0.001$ & $>100$ \\
\hline 9c & $36.6 \pm 10.2$ & $33.0 \pm 8.6$ & $38.6 \pm 8.0$ & $>100$ \\
\hline 10a & $38.2 \pm 3.6$ & $36.3 \pm 12.5$ & $40.6 \pm 8.8$ & $>100$ \\
\hline 10b & $30.0 \pm 1.4$ & $20.8 \pm 4.3$ & $20.3 \pm 2.8$ & $>100$ \\
\hline 10c & $0.01 \pm 0.002$ & $0.01 \pm 0.004$ & $0.04 \pm 0.01$ & - \\
\hline 12a & $60.06 \pm 8.33$ & $58.03 \pm 12.3$ & $33.1 \pm 10.82$ & - \\
\hline 12b & $0.03 \pm 0.002$ & $0.02 \pm 0.003$ & $0.05 \pm 0.002$ & - \\
\hline 14 & $0.04 \pm 0.008$ & $0.09 \pm 0.008$ & $0.09 \pm 0.007$ & $>100$ \\
\hline Doxorubicin & & & \\
\hline
\end{tabular}

\section{EXPERIMENTAL}

\section{Chemistry}

All melting points were determined on an Electrothermal digital melting point apparatus and are uncorrected. IR spectra ( $\mathrm{KBr}$ discs) were recorded on a FTIR plus 460 or Pyeunicam SP-1000 spectrophotometer. ${ }^{1} \mathrm{H}$ NMR spectra were recorded with Mercury-300BB (300 MHz) (Cairo University) instrument in DMSO- $d_{6}$ as solvent using TMS as internal standard and chemical shifts are expressed as $\delta$ ppm. ${ }^{13} \mathrm{C}$-NMR spectra were recorded in DMSO. Analytical data were obtained from the micro analytical data unit at Cairo University and were performed on Vario El III Elemental CHNS analyzer.

N-(3-Cyano-4,5,6,7-tetrahydrobenzo[b]thiophen-2-yl)-3-oxobutanamide (5)

Ethyl acetoacetate $(13.0 \mathrm{~g}, 0.1 \mathrm{~mol})$ was heated till $140{ }^{\circ} \mathrm{C}$ then compound $3(17.8 \mathrm{~g}, 0.1 \mathrm{~mol})$ was added with continuous heating till the temperature reach $125^{\circ} \mathrm{C}$ at this moment the whole reaction mixture is heated under reflux for $20 \mathrm{~min}$. The reaction mixture was allowed to cool overnight and the formed solid product was filtered, dried and crystallized from ethanol to give compound $\mathbf{5}$.

General procedure for the synthesis of the pyran derivatives $7 a-c$

To a solution of compound $5(2.62 \mathrm{~g}, 0.01 \mathrm{~mol})$ in 1,4-dioxane $(40 \mathrm{~mL})$ containing triethyl amine $(0.50 \mathrm{~mL})$ any of benzaldehyde $(1.06 \mathrm{~g}, 0.01 \mathrm{~mol})$, 4-chlorobenzaldehyde $(1.41 \mathrm{~g}, 0.01$ mol) or 4-methoxybenzaldehyde $(1.36 \mathrm{~g}, 0.01 \mathrm{~mol})$ and malononitrile $(0.66 \mathrm{~g}, 0.01 \mathrm{~mol})$ was added. The reaction mixture, in each case, was heated under reflux for $2 \mathrm{~h}$ then left to cool. The formed solid product was collected by filtration. 
2-(5-Acetyl-4-phenyl-4H-pyran-2-ylamino)-4,5,6,7-tetrahydrobenzo[b]thiophene-3-carbonitril (7a). Yield: 83\%; m.p.: 125-128 ${ }^{\circ} \mathrm{C}$; IR $\left(\mathrm{KBr}, \mathrm{cm}^{-1}\right)$ v: $3477-3320\left(\mathrm{NH}_{2}, \mathrm{NH}\right), 3055(\mathrm{CH}$ aromatic), 2936-2850 ( $\mathrm{CH}$ aliphatic), 2223, $2220(2 \mathrm{CN}), 1689(\mathrm{CO}) ;{ }^{1} \mathrm{H}$ NMR (DMSO- $\left.d_{6}\right) \delta$ : 1.73-2.46 (m, 8H, 4CH $), 3.19\left(\mathrm{~s}, 3 \mathrm{H}, \mathrm{CH}_{3}\right), 4.49\left(\mathrm{~s}, 2 \mathrm{H}, \mathrm{D}_{2} \mathrm{O}\right.$ exchangeable, $\left.\mathrm{NH}_{2}\right), 6.01(\mathrm{~s}, 1 \mathrm{H}$, pyran $\mathrm{H}-4), 7.28-7.39\left(\mathrm{~m}, 5 \mathrm{H}, \mathrm{C}_{6} \mathrm{H}_{5}\right), 8.63\left(\mathrm{~s}, 1 \mathrm{H}, \mathrm{NH}, \mathrm{D}_{2} \mathrm{O}\right.$ exchangeable); ${ }^{13} \mathrm{C}$ NMR (DMSO$\left.d_{6}, 400 \mathrm{MHz}\right): 22.8\left(\mathrm{CH}_{3}\right), 29.4,30.5,33.2,38.6\left(4 \mathrm{CH}_{2}\right), 88.3$ (pyran C-4), 116.2, $118.3(2 \mathrm{CN})$, $120.3,121.3,121.8,122.4,126.0,128.4,124.2,130.8,133.0,134.2,137.3,139.2$ (benzene, pyran, thiophene C), 165.8 (CO); MS: $m / z$, (\%): $416(22)$. Anal. calcd. for $\mathrm{C}_{23} \mathrm{H}_{20} \mathrm{~N}_{4} \mathrm{O}_{2} \mathrm{~S}(416.5)$ : C, 66.33; H,4.84; N,13.45; S,7.69. Found: C, 66.53; H, 4.92; N, 13.62; S, 7.92.

2-(5-Acetyl-4-chlorophenyl-4H-pyran-2-ylamino)-4,5,6,7-tetrahydrobenzo[b] thiophene-3carbonitril (7b). Yield: $78 \%$; m.p. $85{ }^{\circ} \mathrm{C}$; IR $\left(\mathrm{KBr}, \mathrm{cm}^{-1}\right)$ v: $3473-3343\left(\mathrm{NH}_{2}, \mathrm{NH}\right), 3058(\mathrm{CH}$ aromatic), 2938-2873 (CH aliphatic), 2226, $2221(2 \mathrm{CN}), 1687(\mathrm{CO}), 1630(\mathrm{C}=\mathrm{C}) ;{ }^{1} \mathrm{H}$ NMR $\left(\mathrm{DMSO}-d_{6}\right) \delta: 1.70-2.48\left(\mathrm{~m}, 8 \mathrm{H}, 4 \mathrm{CH}_{2}\right), 3.17\left(\mathrm{~s}, 3 \mathrm{H}, \mathrm{CH}_{3}\right), 4.48\left(\mathrm{~s}, 2 \mathrm{H}, \mathrm{D}_{2} \mathrm{O}\right.$ exchangeable, $\left.\mathrm{NH}_{2}\right), 6.04(\mathrm{~s}, 1 \mathrm{H}$, pyran $\mathrm{H}-4), 7.23-7.42\left(\mathrm{~m}, 4 \mathrm{H}, \mathrm{C}_{6} \mathrm{H}_{4}\right), 8.68\left(\mathrm{~s}, 1 \mathrm{H}, \mathrm{NH}, \mathrm{D}_{2} \mathrm{O}\right.$ exchangeable); ${ }^{13} \mathrm{C}$ NMR (DMSO- $\left.d_{6}, 400 \mathrm{MHz}\right): 22.8\left(\mathrm{CH}_{3}\right), 29.4,3$ 0.5, 33.2, $38.6\left(4 \mathrm{CH}_{2}\right), 88.3$ (pyran C-4), $116.1,116.4(2 \mathrm{CN}), 120.3,121.3,121.8,122.4,126.0,128.4,124.2,130.8,133.0,134.2,137.3$, 139.2 (benzene, pyran, thiophene C), $165.8(\mathrm{CO})$; MS ( $\mathrm{m} / \mathrm{z}, \%): 450$ (36). Anal. calcd. for $\mathrm{C}_{23} \mathrm{H}_{19} \mathrm{ClN}_{4} \mathrm{O}_{2} \mathrm{~S}$ (450.94): C, 61.26; H, 4.25; N, 12.42; S, 7.11. Found: $\mathrm{C}, 61.09 ; \mathrm{H}, 4.31 ; \mathrm{N}$, $12.69 ; \mathrm{S}, 7.08$.

2-(5-Acetyl-4-methoxyphenyl-4H-pyran-2-ylamino)-4,5,6,7-tetrahydrobenzo[b] thiophene-3carbonitril (7c). Yield: 68\%; m.p. 145-148 ${ }^{\circ} \mathrm{C}$; IR $\left(\mathrm{KBr}, \mathrm{cm}^{-1}\right)$ v: 3495-3318 $\left(\mathrm{NH}_{2}, \mathrm{NH}\right), 3056$ (CH aromatic), 2972-2893 (CH aliphatic), 2224, $2220(2 \mathrm{CN}), 1689(\mathrm{CO}), 1630(\mathrm{C}=\mathrm{C}) ;{ }^{1} \mathrm{H}$ NMR $\left(\right.$ DMSO- $\left._{6}\right) \delta: 1.72-2.40\left(\mathrm{~m}, 8 \mathrm{H}, 4 \mathrm{CH}_{2}\right), 3.11,3.24\left(2 \mathrm{~s}, 6 \mathrm{H}, 2 \mathrm{CH}_{3}\right), 4.44\left(\mathrm{~s}, 2 \mathrm{H}, \mathrm{D}_{2} \mathrm{O}\right.$ exchangeable, $\left.\mathrm{NH}_{2}\right), 6.02(\mathrm{~s}, 1 \mathrm{H}$, pyran $\mathrm{H}-4), 7.28-7.36\left(\mathrm{~m}, 4 \mathrm{H}, \mathrm{C}_{6} \mathrm{H}_{4}\right), 8.60\left(\mathrm{~s}, 1 \mathrm{H}, \mathrm{NH}, \mathrm{D}_{2} \mathrm{O}\right.$ exchangeable); ${ }^{13} \mathrm{C}$ NMR (DMSO- $\left.d_{6}, 400 \mathrm{MHz}\right): 22.6\left(\mathrm{CH}_{3}\right), 29.2,30.5,33.7,38.4\left(4 \mathrm{CH}_{2}\right)$, $32.12\left(\mathrm{CH}_{3}\right), 88.6$ (pyran C-4), 115.8, $116.2(2 \mathrm{CN}), 120.6,122.0,122+.2,122.4,125.3,127.0$, $128.3,130.3,133.3,135.0,136.2,139.0$ (benzene, pyran, thiophene C), 165.9 (CO); MS $(\mathrm{m} / \mathrm{z}$, \%): 446 (22). Anal. calcd. for $\mathrm{C}_{24} \mathrm{H}_{22} \mathrm{~N}_{4} \mathrm{O}_{3} \mathrm{~S}$ (446.52): C, 64.56; H, 4.97; N, 12.55; S, 7.18. Found: C, 64.25; H, 4.77; N, 12.73; S, 7.16.

General procedure for the synthesis of the pyridine derivatives $\mathbf{8 a}-\mathbf{c}$

To a solution of compound $5(2.62 \mathrm{~g}, 0.01 \mathrm{~mol})$ in 1,4-dioxane $(40 \mathrm{~mL})$ containing ammonium acetate $(0.50 \mathrm{~mL})$ any of benzaldehyde $(1.06 \mathrm{~g}, 0.01 \mathrm{~mol}), 4$-chlorobenzaldehyde $(1.41 \mathrm{~g}, 0.01$ mol) or 4-methoxybenzaldehyde $(1.36 \mathrm{~g}, 0.01 \mathrm{~mol})$ malononitrile $(0.66 \mathrm{~g}, 0.01 \mathrm{~mol})$ was added. The reaction mixture, in each case, was heated under reflux for $4 \mathrm{~h}$ then left to cool. The formed solid product was collected by filtration.

2-(5-Acetyl-1,4-dihydro-4-phenylpyridin-2-ylamino)-4,5,6,7-tetrahydrobenzo [b]thiophene-3carbonitril (8a). Yield: 77\%; m.p. 102-104 ${ }^{\circ} \mathrm{C}$; IR $\left(\mathrm{KBr}, \mathrm{cm}^{-1}\right)$ v: 3483-3316 $\left(\mathrm{NH}_{2}, 2 \mathrm{NH}\right), 3053$ (CH aromatic), 2948-2862 (CH aliphatic), 2224, $2220(2 \mathrm{CN}), 1687(\mathrm{CO}), 1633(\mathrm{C}=\mathrm{C}) ;{ }^{1} \mathrm{H}$ NMR $\left(\right.$ DMSO- $\left.d_{6}\right) \delta: 1.70-2.43\left(\mathrm{~m}, 8 \mathrm{H}, 4 \mathrm{CH}_{2}\right), 3.16\left(\mathrm{~s}, 3 \mathrm{H}, \mathrm{CH}_{3}\right), 4.44\left(\mathrm{~s}, 2 \mathrm{H}, \mathrm{D}_{2} \mathrm{O}\right.$ exchangeable, $\left.\mathrm{NH}_{2}\right), 6.01(\mathrm{~s}, 1 \mathrm{H}$, pyran $\mathrm{H}-4), 7.25-7.39\left(\mathrm{~m}, 5 \mathrm{H}, \mathrm{C}_{6} \mathrm{H}_{5}\right), 8.60,8.72\left(2 \mathrm{~s}, 2 \mathrm{H}, 2 \mathrm{NH}, \mathrm{D}_{2} \mathrm{O}\right.$ exchangeable); ${ }^{13} \mathrm{C}$ NMR (DMSO- $\left.d_{6}, 400 \mathrm{MHz}\right): 22.7\left(\mathrm{CH}_{3}\right), 29.0,30.8,32.9,38.9\left(4 \mathrm{CH}_{2}\right), 88.2$ (pyran C-4), 116.2, 116.9 (2CN), 120.6, 121.0, 121.6, 122.0, 124.7, 127.9, 128.3, 130.5, 133.2, 133.9, 137.7, 139.3 (benzene, pyridine, thiophene C), $166.0(\mathrm{CO})$; $\mathrm{MS}(\mathrm{m} / \mathrm{z}, \%): 415$ (18). Anal. calcd. for $\mathrm{C}_{23} \mathrm{H}_{21} \mathrm{~N}_{5} \mathrm{OS}(415.51)$ : C, 66.48; $\mathrm{H}, 5.09 ; \mathrm{N}, 16.85 ; \mathrm{S}, 7.72$. Found: C, 66.50; H, 4.92; N, 16.53; S, 7.92. 
2-(5-Acetyl-1,4-dihydro-4-chloro-phenylpyridin-2-ylamino)-4,5,6,7-tetrahydrobenzo[b] thiophene-3-carbonitril (8b). Yield 79\%; m.p.: 116-1 $19{ }^{\circ} \mathrm{C}$; IR $\left(\mathrm{KBr}, \mathrm{cm}^{-1}\right)$ v: 3488-3323 $\left(\mathrm{NH}_{2}, 2 \mathrm{NH}\right)$, 3058 (CH aromatic), 2938-2873 (CH aliphatic), 2226, $2221(2 \mathrm{CN}), 1687(\mathrm{CO}), 1630(\mathrm{C}=\mathrm{C}) ;{ }^{1} \mathrm{H}$ NMR $\left(\mathrm{DMSO}-d_{6}\right) \delta$ : $1.73-2.45\left(\mathrm{~m}, 8 \mathrm{H}, 4 \mathrm{CH}_{2}\right), 3.14\left(\mathrm{~s}, 3 \mathrm{H}, \mathrm{CH}_{3}\right), 4.45\left(\mathrm{~s}, 2 \mathrm{H}, \mathrm{D}_{2} \mathrm{O}\right.$ exchangeable, $\left.\mathrm{NH}_{2}\right), 6.02$ (s, $1 \mathrm{H}$, pyran $\left.\mathrm{H}-4\right), 7.26-7.45\left(\mathrm{~m}, 4 \mathrm{H}, \mathrm{C}_{6} \mathrm{H}_{4}\right), 8.33,8.65(2 \mathrm{~s}, 2 \mathrm{H}$, $2 \mathrm{NH}, \mathrm{D}_{2} \mathrm{O}$ exchangeable); ${ }^{13} \mathrm{C}$ NMR (DMSO-d $\left.d_{6}, 400 \mathrm{MHz}\right): 22.4\left(\mathrm{CH}_{3}\right), 28.3,31.8,32.8,37.1$ $\left(4 \mathrm{CH}_{2}\right), 88.6$ (pyran C-4), 116.0, $116.3(2 \mathrm{CN}), 120.3,121.6,121.8,122.2,124.8,127.0,128.3$, 131.9, 132.8, 134.9, 138.5, 139.2 (benzene, pyran, thiophene C), $164.8(\mathrm{CO})$; MS (m/z, \%): 449 (38). Anal. calcd. for $\mathrm{C}_{23} \mathrm{H}_{20} \mathrm{ClN}_{5} \mathrm{OS}$ (449.11): C, 61.39; H, 4.48; N, 15.56; S, 7.13. Found: $\mathrm{C}$, $61.42 ; \mathrm{H}, 4.63 ; \mathrm{N}, 15.36 ; \mathrm{S}, 7.28$.

2-(5-Acetyl-1,4-dihydro-4-methoxy-phenylpyridin-2-ylamino)-4,5,6,7-tetrahydrobenzo[b] thiophene-3-carbonitril $(\boldsymbol{8 c})$. Yield: $83 \%$; m.p.: $140-142{ }^{\circ} \mathrm{C}$; IR $\left(\mathrm{KBr}, \mathrm{cm}^{-1}\right)$ v: $3495-3318\left(\mathrm{NH}_{2}\right.$, $2 \mathrm{NH}), 3056$ (CH aromatic), 2972-2893 (CH aliphatic), 2224, $2220(2 \mathrm{CN}), 1689$ (CO), 1630 $(\mathrm{C}=\mathrm{C}) ;{ }^{1} \mathrm{H}$ NMR $\left(\mathrm{DMSO}-d_{6}\right) \delta: 1.72-2.40\left(\mathrm{~m}, 8 \mathrm{H}, 4 \mathrm{CH}_{2}\right), 3.11,3.24\left(2 \mathrm{~s}, 6 \mathrm{H}, 2 \mathrm{CH}_{3}\right), 4.44(\mathrm{~s}$, $2 \mathrm{H}, \mathrm{D}_{2} \mathrm{O}$ exchangeable, $\left.\mathrm{NH}_{2}\right), 6.02(\mathrm{~s}, 1 \mathrm{H}$, pyran $\mathrm{H}-4), 7.28-7.36\left(\mathrm{~m}, 4 \mathrm{H}, \mathrm{C}_{6} \mathrm{H}_{4}\right), 8.32,8.60(2 \mathrm{~s}$, $2 \mathrm{H}, 2 \mathrm{NH}, \mathrm{D}_{2} \mathrm{O}$ exchangeable); ${ }^{13} \mathrm{C}$ NMR (DMSO- $\left.d_{6}, 400 \mathrm{MHz}\right): 22.6\left(\mathrm{CH}_{3}\right), 29.2,30.5,33.7$, $38.4\left(4 \mathrm{CH}_{2}\right), 32.12\left(\mathrm{CH}_{3}\right), 88.6$ (pyran C-4), 115.8, $116.4(2 \mathrm{CN}), 120.2,122.3,122.8,122.4$, 125.3, 127.8, 129.1, 132.7, 133.6, 135.0, 136.2 (benzene, pyran, thiophene C), 165.6 (CO); MS $(\mathrm{m} / z, \%)$ : 445 (16). Anal. calcd. for $\mathrm{C}_{24} \mathrm{H}_{23} \mathrm{~N}_{5} \mathrm{O}_{2} \mathrm{~S}$ (445.54): $\mathrm{C}, 64.70 ; \mathrm{H}, 5.20 ; \mathrm{N}, 15.72 ; \mathrm{S}$, 7.20. Found: C, 64.49; H, 5.26; N, 15.91; S, 7.42.

General procedure for the synthesis of the 5-amino-4-cyanothiophen-3-yl)-4-phenyl-4H-pyran derivatives $\mathbf{9 a - c}$

To a solution of any of compound $7 \mathbf{a}(4.15 \mathrm{~g}, 0.01 \mathrm{~mol}), 7 \mathbf{b}(4.50 \mathrm{~g}, 0.01 \mathrm{~mol})$ or $7 \mathbf{c}(4.46 \mathrm{~g}$, $0.01 \mathrm{~mol})$ in 1,4-dioxane $(40 \mathrm{~mL})$ containing triethyl amine $(1.50 \mathrm{~mL})$ elemental sulfur $(0.32 \mathrm{~g}$, $0.01 \mathrm{~mol})$ and malononitrile $(0.66 \mathrm{~g}, 0.01 \mathrm{~mol})$ were added. The whole reaction mixture was heated under reflux for $2 \mathrm{~h}$ then poured onto ice/water containing few drops of hydrochloric acid and the formed solid product was collected by filtration.

2-Amino-5-(5-amino-4-cyanothiophen-3-yl)-6-((3-cyano-4,5,6,7-tetrahydrobenzo-[b] thiophen2-yl)amino)-4-phenyl-4H-pyran-3-carbonitrile (9a). Yield: 70\%; m.p.: 230-235 ${ }^{\circ} \mathrm{C}$; IR ( $\mathrm{KBr}$, $\left.\mathrm{cm}^{-1}\right) v: 3453-3341\left(2 \mathrm{NH}_{2}, \mathrm{NH}\right), 3053$ (CH aromatic), 2983, 2851 (CH aliphatic), 2228, 2223$2220(3 \mathrm{CN}), 1636(\mathrm{C}=\mathrm{C}) ;{ }^{1} \mathrm{H}$ NMR $\left(\mathrm{DMSO}-d_{6}\right) \delta: 1.43-2.51\left(\mathrm{~m}, 8 \mathrm{H}, 4 \mathrm{CH}_{2}\right), 4.38,4.70(2 \mathrm{~s}, 4 \mathrm{H}$, $\mathrm{D}_{2} \mathrm{O}$ exchangeable, $\left.2 \mathrm{NH}_{2}\right), 5.96(\mathrm{~s}, 1 \mathrm{H}$, thiophene $\mathrm{H}-5), 6.04(\mathrm{~s}, 1 \mathrm{H}$, pyran $\mathrm{H}-4), 7.28-7.46(\mathrm{~m}$, $\left.5 \mathrm{H}, \mathrm{C}_{6} \mathrm{H}_{5}\right), 8.39$ (s, $1 \mathrm{H}, \mathrm{NH}, \mathrm{D}_{2} \mathrm{O}$ exchangeable); ${ }^{13} \mathrm{C}$ NMR (DMSO-d $d_{6}, 400 \mathrm{MHz}$ ): 29.3, 30.5, 32.9, $38.3\left(4 \mathrm{CH}_{2}\right), 88.6$ (pyran C-4), 115.7, 116.0, $116.3(3 \mathrm{CN}), 119.5,120.8,121.3,122.9$, $123.3,126.9,127.5,130.8,132.6,133.5,137.7,138.5,139.7,140.6,142.9,145.2$ (benzene, pyran, two thiophene C); MS (m/z, \%): 496 (42). Anal. calcd. for $\mathrm{C}_{26} \mathrm{H}_{20} \mathrm{~N}_{6} \mathrm{OS}_{2}$ (496.61): $\mathrm{C}$, 62.88; H, 4.06; N, 16.92; S, 12.91. Found: C, 62.94; H, 4.33; N, 17.18; S, 13.07 .

2-Amino-5-(5-amino-4-cyanothiophen-3-yl)-4-(4-chlorophenyl)-6-((3-cyano-4,5,6,7-tetrahydrobenzo[b] thiophen-2-yl)amino)-4H-pyran-3-carbonitrile (9b). Yield 84\%; m.p.: 216-218 ${ }^{\circ} \mathrm{C}$; IR $\left(\mathrm{KBr}, \mathrm{cm}^{-1}\right)$ v: 3481-3342 (2NH $\left.2, \mathrm{NH}\right), 3052$ (CH aromatic), 2948-2881 (CH aliphatic), 2226, $2223-2220(3 \mathrm{CN}), 1689(\mathrm{CO}), 1632(\mathrm{C}=\mathrm{C}) ;{ }^{1} \mathrm{H}$ NMR $\left(\mathrm{DMSO}-d_{6}\right) \delta: 1.76-2.48\left(\mathrm{~m}, 8 \mathrm{H}, 4 \mathrm{CH}_{2}\right)$, 4.36, $4.90\left(2 \mathrm{~s}, 4 \mathrm{H}, \mathrm{D}_{2} \mathrm{O}\right.$ exchangeable, $\left.2 \mathrm{NH}_{2}\right), 5.95(\mathrm{~s}, 1 \mathrm{H}$, thiophene $\mathrm{H}-5), 6.04(\mathrm{~s}, 1 \mathrm{H}$, pyran $\mathrm{H}-4), 7.30-7.48\left(\mathrm{~m}, 4 \mathrm{H}, \mathrm{C}_{6} \mathrm{H}_{4}\right), 8.29\left(\mathrm{~s}, 1 \mathrm{H}, \mathrm{NH}, \mathrm{D}_{2} \mathrm{O}\right.$ exchangeable); ${ }^{13} \mathrm{C}$ NMR (DMSO- $d_{6}, 400$ MHz): 28.1, 31.4, 31.9, $35.8\left(4 \mathrm{CH}_{2}\right), 88.9$ (pyran C-4), 116.1, 116.7, $117.3(3 \mathrm{CN}), 120.8,121.9$, $122.4,123.8,125.3,126.9,128.8,130.4,131.3,132.7,136.5,137.9,138.0,140.8,142.8,143.6$ 
(benzene, pyran, two thiophene C); MS (m/z, \%): 531 (32). Anal. calcd. for $\mathrm{C}_{26} \mathrm{H}_{19} \mathrm{ClN}_{6} \mathrm{OS}_{2}$ (531.05): C, 58.80; H, 3.61; N, 15.83; S, 12.08. Found: C, 59.28; H, 3.94; N, 16.33; S, 11.94.

2-Amino-5-(5-amino-4-cyanothiophen-3-yl)-4-(4-chlorophenyl)-6-((3-cyano-4,5,6,7-tetrahydrobenzo[b] thiophen-2-yl)amino)-4H-pyran-3-carbonitrile (9c). Yield $73 \%$; m.p.: $>300{ }^{\circ} \mathrm{C}$; IR $\left(\mathrm{KBr}, \mathrm{cm}^{-1}\right)$ v: $3472-3321\left(2 \mathrm{NH}_{2}, \mathrm{NH}\right), 3058$ (CH aromatic), 2973, 2890 (CH aliphatic), 2227, 2222-2220 (3CN), $1688(\mathrm{CO}), 1633(\mathrm{C}=\mathrm{C}) ;{ }^{1} \mathrm{H}$ NMR $\left(\mathrm{DMSO}-d_{6}\right) \delta: 1.68-2.42\left(\mathrm{~m}, 8 \mathrm{H}, 4 \mathrm{CH}_{2}\right)$, $3.24\left(\mathrm{~s}, 3 \mathrm{H}, \mathrm{CH}_{3}\right), 4.42,4.57\left(2 \mathrm{~s}, 4 \mathrm{H}, \mathrm{D}_{2} \mathrm{O}\right.$ exchangeable, $\left.2 \mathrm{NH}_{2}\right), 6.08$ (s, $1 \mathrm{H}$, pyran $\left.\mathrm{H}-4\right), 6.22$ (s, 1H, thiophene $\mathrm{H}-5), 7.28-7.36\left(\mathrm{~m}, 4 \mathrm{H}, \mathrm{C}_{6} \mathrm{H}_{4}\right), 8.34\left(\mathrm{~s}, 1 \mathrm{H}, \mathrm{NH}, \mathrm{D}_{2} \mathrm{O}\right.$ exchangeable $) ;{ }^{13} \mathrm{C}$ NMR (DMSO- $\left.d_{6}, 400 \mathrm{MHz}\right): 28.6,31.6,32.9,35.8\left(4 \mathrm{CH}_{2}\right), 32.3\left(\mathrm{CH}_{3}\right), 88.7$ (pyran C-4), 115.6, 116.5, $116.8(3 \mathrm{CN}), 119.8,121.6,122.4,123.9,124.9,127.5,128.5,130.4,132.3,132.9$, 134.3, 135.7, 136.2, 140.9, 141.8, 143.9 (benzene, pyran, twothiophene C); MS ( $\mathrm{m} / \mathrm{z}, \%): 526$ (26). Anal. calcd. for $\mathrm{C}_{27} \mathrm{H}_{22} \mathrm{~N}_{6} \mathrm{O}_{2} \mathrm{~S}_{2}$ (526.63): C, 61.58; H, 4.21; N, 15.98; S, 12.18. Found: C, $61.83 ; \mathrm{H}, 4.47 ; \mathrm{N}, 16.31 ; \mathrm{S}, 12.22$.

General procedure for the synthesis of the 5-amino-4-cyanothiophen-3-yl)-4-phenyl-4Hpyridine derivatives $10 a-c$

To a solution of any of compound $\mathbf{8 a}(4.15 \mathrm{~g}, 0.01 \mathrm{~mol}), \mathbf{8 b}(4.49 \mathrm{~g}, 0.01 \mathrm{~mol})$ or $\mathbf{8 c}(4.45 \mathrm{~g}$, $0.01 \mathrm{~mol})$ in 1,4-dioxane $(40 \mathrm{~mL})$ containing triethyl amine $(1.50 \mathrm{~mL})$ elemental sulfur $(0.32 \mathrm{~g}$, $0.01 \mathrm{~mol})$ and malononitrile $(0.66 \mathrm{~g}, 0.01 \mathrm{~mol})$ were added. The whole reaction mixture was heated under reflux for $2 \mathrm{~h}$ then poured onto ice/water containing few drops of hydrochloric acid and the formed solid product was collected by filtration.

2-Amino-5-(5-amino-4-cyanothiophen-3-yl)-6-((3-cyano-4,5,6,7-tetrahydrobenzo-[b] thiophen2-yl)amino)-4-phenyl-1,4-dihydropyridine-3-carbonitrile (10a). Yield: 83\%; m.p. $145^{\circ} \mathrm{C}$; IR $\left(\mathrm{KBr}, \mathrm{cm}^{-1}\right)$ v: 3463-3329 (2NH $\left.2,2 \mathrm{NH}\right), 3056(\mathrm{CH}$ aromatic), 2967-2851 (CH aliphatic), 2227, 2222-2220 (3CN), $1631(\mathrm{C}=\mathrm{C})$; ${ }^{1} \mathrm{H}$ NMR (DMSO- $\left.d_{6}\right) \delta: 1.48-2.39\left(\mathrm{~m}, 8 \mathrm{H}, 4 \mathrm{CH}_{2}\right), 4.43,4.72$ $\left(2 \mathrm{~s}, 4 \mathrm{H}, \mathrm{D}_{2} \mathrm{O}\right.$ exchangeable, $\left.2 \mathrm{NH}_{2}\right), 5.99(\mathrm{~s}, 1 \mathrm{H}$, thiophene $\mathrm{H}-5), 6.03$ (s, 1H, pyridine $\left.\mathrm{H}-4\right)$, 7.22-7.42 (m, 5H, $\left.\mathrm{C}_{6} \mathrm{H}_{5}\right), 8.54,8.65\left(2 \mathrm{~s}, 2 \mathrm{H}, 2 \mathrm{NH}, \mathrm{D}_{2} \mathrm{O}\right.$ exchangeable); ${ }^{13} \mathrm{C}$ NMR (DMSO- $d_{6}$, $400 \mathrm{MHz}$ ): 29.0, 30.8, 32.9, $38.9\left(4 \mathrm{CH}_{2}\right), 88.2$ (pyridine C-4), 116.1, 116.3, $116.8(3 \mathrm{CN}), 120.6$, 121.0, 121.6, 122.0, 124.7, 127.9, 128.3, 130.5, 133.2, 133.9, 137.7, 138.0, 139.3, 141.3, 144.2, 146.8 (benzene, pyridine, two thiophene C); MS (m/z, 495 (33). Anal. calcd. for $\mathrm{C}_{26} \mathrm{H}_{21} \mathrm{~N}_{7} \mathrm{~S}_{2}$ (495.62): C, 63.01; H, 4.27; N, 19.78; S, 12.94. Found: C, 62.88; H, 4.51; N, 19.49; S, 13.19.

2-Amino-5-(5-amino-4-cyanothiophen-3-yl)-6-((3-cyano-4,5,6,7-tetrahydrobenzo-[b] thiophen2-yl)amino)-4-chlorophenyl-1,4-dihydropyridine-3-carbonitrile (10b). Yield 88\%; m.p. 180-182 ${ }^{\circ} \mathrm{C}$; IR $\left(\mathrm{KBr}, \mathrm{cm}^{-1}\right)$ v: 3463-3313 $\left(2 \mathrm{NH}_{2}, 2 \mathrm{NH}\right), 3054$ ( $\mathrm{CH}$ aromatic), 2942-2870 (CH aliphatic), 2224, 2222-2220 (3CN), $1630(\mathrm{C}=\mathrm{C}) ;{ }^{1} \mathrm{H}$ NMR (DMSO-d 6 ) $\delta: 1.73-2.45\left(\mathrm{~m}, 8 \mathrm{H}, 4 \mathrm{CH}_{2}\right), 4.29$, $4.45\left(2 \mathrm{~s}, 4 \mathrm{H}, \mathrm{D}_{2} \mathrm{O}\right.$ exchangeable, $\left.2 \mathrm{NH}_{2}\right), 5.99(\mathrm{~s}, 1 \mathrm{H}$, thiophene $\mathrm{H}-5), 6.02$ (s, $1 \mathrm{H}$, pyridine $\mathrm{H}-$ 4), 7.26-7.43 (m, 4H, $\left.\mathrm{C}_{6} \mathrm{H}_{4}\right), 8.33,8.65\left(2 \mathrm{~s}, 2 \mathrm{H}, 2 \mathrm{NH}, \mathrm{D}_{2} \mathrm{O}\right.$ exchangeable); ${ }^{13} \mathrm{C}$ NMR (DMSO$\left.d_{6}, 400 \mathrm{MHz}\right): 28.3,31.8,32.8,37.1\left(4 \mathrm{CH}_{2}\right), 88.6$ (pyridine $\left.\mathrm{C}-4\right), 116.0,116.4,116.9(3 \mathrm{CN})$, $120.3,121.6,121.8,122.2,124.8,127.0,128.3,131.9,132.8,134.9,138.3,138.5,139.2141 .2$, 142.8, 143.9 (benzene, pyridine, two thiophene C); MS ( $\mathrm{m} / \mathrm{z}, 530$ (42). Anal. calcd. for $\mathrm{C}_{26} \mathrm{H}_{20} \mathrm{ClN}_{7} \mathrm{~S}_{2}$ (530.07): C, 58.91; H, 3.80; N, 18.50; S, 12.10. Found: C, 59.09; H, 3.66; N, $18.83 ; \mathrm{S}, 11.89$.

2-Amino-5-(5-amino-4-cyanothiophen-3-yl)-6-((3-cyano-4,5,6,7-tetrahydrobenzo-[b] thiophen2-yl)amino)-4-methoxyphenyl-1,4-dihydropyridine-3-carbonitrile (10c). Yield 68\%; m.p. 110 ${ }^{\circ} \mathrm{C}$; IR $\left(\mathrm{KBr}, \mathrm{cm}^{-1}\right)$ v: 3488-3342 $\left(2 \mathrm{NH}_{2}, 2 \mathrm{NH}\right), 3054(\mathrm{CH}$ aromatic), 2960, 2892 (CH aliphatic), 2225, 2222-2220 (3CN), $1633(\mathrm{C}=\mathrm{C}) ;{ }^{1} \mathrm{H}$ NMR (DMSO- $\left.d_{6}\right) \delta: 1.68-2.42\left(\mathrm{~m}, 8 \mathrm{H}, 4 \mathrm{CH}_{2}\right), 3.26(\mathrm{~s}$, 
$\left.3 \mathrm{H}, \mathrm{CH}_{3}\right), 4.46,4.69\left(2 \mathrm{~s}, 4 \mathrm{H}, \mathrm{D}_{2} \mathrm{O}\right.$ exchangeable, $\left.2 \mathrm{NH}_{2}\right), 6.04(\mathrm{~s}, 1 \mathrm{H}$, pyridine $\mathrm{H}-4), 6.11$ (s, $1 \mathrm{H}$, thiophene $\mathrm{H}-5), 7.25-7.39\left(\mathrm{~m}, 4 \mathrm{H}, \mathrm{C}_{6} \mathrm{H}_{5}\right), 8.31,8.63\left(2 \mathrm{~s}, 2 \mathrm{H}, 2 \mathrm{NH}, \mathrm{D}_{2} \mathrm{O}\right.$ exchangeable $) ;{ }^{13} \mathrm{C}$ NMR (DMSO- $\left.d_{6}, 400 \mathrm{MHz}\right): 29.0,30.8,33.4,36.7\left(4 \mathrm{CH}_{2}\right), 31.8\left(\mathrm{CH}_{3}\right), 88.9$ (pyridine C-4), $115.7,116.1,116.3(3 \mathrm{CN}), 120.2,122.3,122.8,122.4,125.3,127.8,129.1,130.9,132.7,133.6$, 135.0, 136.2, 138.1, 140.4, 142.5, 143.2 (benzene, pyridine, two thiophene C); MS $(\mathrm{m} / \mathrm{z}, \%)$ : 525 (26). Anal. calcd. for $\mathrm{C}_{27} \mathrm{H}_{23} \mathrm{~N}_{7} \mathrm{OS}_{2}$ (525.65): $\mathrm{C}, 61.69 ; \mathrm{H}, 4.41 ; \mathrm{N}, 18.65 ; \mathrm{S}, 12.20$. Found: C, $62.33 ; \mathrm{H}, 4.68 ; \mathrm{N}, 18.89 ; \mathrm{S}, 12.41$.

General procedure for the synthesis of the thiophene derivatives $\mathbf{1 2 a} \boldsymbol{a} \mathbf{b}$

To a solution of compound $5(2.62 \mathrm{~g}, 0.01 \mathrm{~mol})$, in 1,4-dioxane $(40 \mathrm{~mL})$ containing triethyl amine $(1.50 \mathrm{~mL})$ elemental sulfur $(0.32 \mathrm{~g}, 0.01 \mathrm{~mol})$ and either of malononitrile $(0.66 \mathrm{~g}, 0.01$ mol) or ethyl cyanoacetate $(1.07 \mathrm{~g}, 0.01 \mathrm{~mol})$ were added. The whole reaction mixture was heated under reflux for $4 \mathrm{~h}$ then poured onto ice/water containing few drops of hydrochloric acid and the formed solid product was collected by filtration.

2-(5-Amino-4-cyanothiophen-3-yl)-N-(3-cyano-4,5,6,7-tetrahydrobenzo-[b] thiophen-2-yl)acetamide $(\mathbf{1 2 a})$. Yield $80 \%$; m.p. $120{ }^{\circ} \mathrm{C}$; IR $\left(\mathrm{KBr}, \mathrm{cm}^{-1}\right)$ v: $3459-3328\left(\mathrm{NH}_{2}, \mathrm{NH}\right), 3053(\mathrm{CH}$ aromatic), 2972, 2869 (CH aliphatic), 2224, $2220(2 \mathrm{CN}), 1703(\mathrm{CO}), 1630(\mathrm{C}=\mathrm{C}) ;{ }^{1} \mathrm{H}$ NMR $\left(\mathrm{DMSO}-d_{6}\right) \delta: 1.65-2.41\left(\mathrm{~m}, 8 \mathrm{H}, 4 \mathrm{CH}_{2}\right), 3.88\left(\mathrm{~s}, 2 \mathrm{H}, \mathrm{CH}_{2}\right), 4.42\left(\mathrm{~s}, 2 \mathrm{H}, \mathrm{D}_{2} \mathrm{O}\right.$ exchangeable, $\left.\mathrm{NH}_{2}\right), 6.18(\mathrm{~s}, 1 \mathrm{H}$, thiophene $\mathrm{H}-5), 8.33\left(\mathrm{~s}, 1 \mathrm{H}, \mathrm{NH}, \mathrm{D}_{2} \mathrm{O}\right.$ exchangeable); ${ }^{13} \mathrm{C}$ NMR (DMSO- $d_{6}$, $400 \mathrm{MHz}): 29.0,30.5,33.1,36.9\left(4 \mathrm{CH}_{2}\right), 43.8\left(\mathrm{CH}_{2}\right), 116.3,116.6(2 \mathrm{CN}), 130.8,133.1,133.6$, 137.1, 138.1, 142.9, 143.6 (two thiophene C), 166.2 (CO); MS ( $\mathrm{m} / \mathrm{z}, \%)$ : 342 (48). Anal. calcd. for $\mathrm{C}_{16} \mathrm{H}_{14} \mathrm{~N}_{4} \mathrm{OS}_{2}$ (342.44): C, 56.12; H, 4.12; N, 16.36; S, 18.73. Found: C, 56.08; H, 4.33; N, $16.59 ; \mathrm{S}, 18.92$.

Ethyl-2-amino-4-(2-((3-cyano-4,5,6,7-tetrahydrobenzo[b] thiophen-2-yl)amino)-2-oxoethyl)thiophene-3-carboxylate (12b). Yield 67\%; m.p. $110{ }^{\circ} \mathrm{C}$; IR $\left(\mathrm{KBr}, \mathrm{cm}^{-1}\right)$ v: 3483-3330 $\left(\mathrm{NH}_{2}, \mathrm{NH}\right)$, 3054 (CH aromatic), 2983, 2863 (CH aliphatic), $2220(\mathrm{CN}), 1705,1688(2 \mathrm{CO}), 1632(\mathrm{C}=\mathrm{C})$; ${ }^{1} \mathrm{H}$ NMR (DMSO- $\left.d_{6}\right) \delta: 1.16\left(\mathrm{t}, 3 \mathrm{H}, \mathrm{J}=6.95 \mathrm{~Hz}, \mathrm{CH}_{3}\right), 1.62-2.48\left(\mathrm{~m}, 8 \mathrm{H}, 4 \mathrm{CH}_{2}\right), 3.92(\mathrm{~s}, 2 \mathrm{H}$, $\left.\mathrm{CH}_{2}\right), 4.21\left(\mathrm{q}, 2 \mathrm{H}, \mathrm{J}=6.95 \mathrm{~Hz}, \mathrm{CH}_{2}\right), 4.49\left(\mathrm{~s}, 2 \mathrm{H}, \mathrm{D}_{2} \mathrm{O}\right.$ exchangeable, $\left.\mathrm{NH}_{2}\right), 6.18(\mathrm{~s}, 1 \mathrm{H}$, thiophene $\mathrm{H}-5), 8.33$ (s, $1 \mathrm{H}, \mathrm{NH}, \mathrm{D}_{2} \mathrm{O}$ exchangeable); ${ }^{13} \mathrm{C}$ NMR (DMSO- $d_{6}, 400 \mathrm{MHz}$ ): 14.2 (ester $\left.\mathrm{CH}_{3}\right), 29.2,30.9,33.3,36.9\left(4 \mathrm{CH}_{2}\right), 43.6\left(\mathrm{CH}_{2}\right), 53.8$ (ester $\left.\mathrm{CH}_{2}\right), 116.4(\mathrm{CN}), 130.2$, 133.3, 133.9, 135.8, 137.4, 142.6, 144.2 (two thiophene C), 166.8, 168.3 (2CO); MS ( $\mathrm{m} / \mathrm{z}, \%)$ : 389 (31). Anal. calcd. for $\mathrm{C}_{18} \mathrm{H}_{19} \mathrm{~N}_{3} \mathrm{O}_{3} \mathrm{~S}_{2}$ (389.49): C, 55.51; H, 4.92; N, 10.79; S, 16.47. Found: C, 55.39; H, 5.18; N, 11.09; S, 16.62.

3-Oxo-N-(3-(4-oxo-4,5-dihydrothiazol-2-yl)-4,5,6,7-tetrahydrobenzo-[b] thiophen-2-yl)butanemide (14). To a solution of compound $5(2.62 \mathrm{~g}, 0.01 \mathrm{~mol})$ in acetic acid $(50 \mathrm{~mL})$, thioglycollic acid $(0.92 \mathrm{~g}, 0.01 \mathrm{~mol})$ was added. the reaction mixture was heated under reflux for $2 \mathrm{~h}$ then was left to cool. The solid product produced upon pouring onto ice/water mixture was collected by filtration. Yield 58\%; m.p. $140{ }^{\circ} \mathrm{C}$; IR $\left(\mathrm{KBr}, \mathrm{cm}^{-1}\right)$ v: 3495-3321 (NH), 2972, $2829(\mathrm{CH}$ aliphatic), 1708, 1689-1660 (3CO), $1636(\mathrm{C}=\mathrm{C}) ;{ }^{1} \mathrm{H}$ NMR (DMSO- $\left.d_{6}\right) \delta: 2.36\left(\mathrm{~s}, 3 \mathrm{H}, \mathrm{CH}_{3}\right)$, $1.59-2.45\left(\mathrm{~m}, 8 \mathrm{H}, 4 \mathrm{CH}_{2}\right), 3.96\left(\mathrm{~s}, 2 \mathrm{H}, \mathrm{CH}_{2}\right), 5.30\left(\mathrm{~s}, 2 \mathrm{H}\right.$, thiazole $\left.\mathrm{CH}_{2}\right), 8.33\left(\mathrm{~s}, 1 \mathrm{H}, \mathrm{NH}, \mathrm{D}_{2} \mathrm{O}\right.$ exchangeable); ${ }^{13} \mathrm{C}$ NMR (DMSO- $\left.d_{6}, 400 \mathrm{MHz}\right): 29.6,29.6,32.8,34.9\left(4 \mathrm{CH}_{2}\right), 45.2\left(\mathrm{CH}_{2}\right), 89.2$ (thiazole $\mathrm{CH}_{2}$ ), 132.6, 136.2, 142.9, 144.9 (thiophene $\mathrm{C}$ ), 164.8, 163.6, 169.0 (3CO), 170.3 $(\mathrm{C}=\mathrm{N})$; $\mathrm{MS}(\mathrm{m} / \mathrm{z}, \%): 336$ (24). Anal. calcd. for $\mathrm{C}_{15} \mathrm{H}_{16} \mathrm{~N}_{2} \mathrm{O}_{3} \mathrm{~S}_{2}$ (336.43): C, 53.55; $\mathrm{H}, 4.79 ; \mathrm{N}$, 8.33; S, 19.06. Found: C, 53.80; H, 4.88; N, 8.58; S, 18.79. 


\section{CONCLUSION}

The newly synthesized thiophene derivatives were obtained in optimal yields and their structures were confirmed using the analytical and spectral tools. The antitumor evaluations of the synthesized compounds against the three cancer cell lines MCF-7, NCI-H460 and SF-268 showed that compounds $9 \mathbf{a}, \mathbf{9 c}, \mathbf{1 2 a}$ and $\mathbf{1 4}$ have the highest potencies against the three cancer cell lines among the tested compounds.

\section{REFERENCES}

1. Basoglu, A.; Dirkmann, S.; Golpayegani, N.Z.; Silke Vortherms, S.; Tentrop, J.; Nowottnik, D.; Prinz, H.; Frohlich, R.; Müller, K. Oxadiazole-substituted naphtho[2,3-b]thiophene-4,9diones as potent inhibitors of keratinocyte hyperproliferation. Structureactivity relationships of the tricyclic quinone skeleton and the oxadiazole substituent. Eur. J. Med. Chem. 2017, 134, 119-132.

2. Fakhr, I.M.I.; Radwan, M.A.A.; El-Batran, S.; Abd El-Salam, O.M.E.; Siham M. ElShenawy, S.M. Synthesis and pharmacological evaluation of 2-substituted benzo[b]thiophenes as anti-inflammatory and analgesic agents. Eur. J. Med. Chem. 2009, 44, 1718-1725.

3. Kulandasamy, R.; Adhikari, A.V.; Stables, J.P. A new class of anticonvulsants possessing 6 Hz activity: 3,4-Dialkyloxy thiophene bishydrazones. Eur. J. Med. Chem. 2009, 44, 43764384.

4. Mohareb, R.M.; Mayssoune Y. Zaki, M.Y.; Nermeen S. Abbas, N.S. Synthesis, antiinflammatory and anti-ulcer evaluations of thiazole, thiophene, pyridine and pyran derivatives derived from androstenedione. Steroids 2015, 98, 80-91.

5. Zhang, L.; Chen, C.J.; Chen, J.; Zhao, Q.Q.; Ya Li, Y.; Gao, K. Thiophene acetylenes and furanosesquiterpenes from Xanthopappus subacaulis and their antibacterial activities. Phytochemistry 2014, 106, 134-140.

6. Khalil, A.M.; Berghot, M.A.; Gouda, M.A. Synthesis and antibacterial activity of some new thiazole and thiophene derivatives. Eur. J. Med. Chem. 2009, 44, 4434-4440.

7. Amr, A.E.; Sherif, M.H.; Assy, M.G.; Al-Omar, M.A.; Ragab, I. Antiarrhythmic, serotonin antagonist and antianxiety activities of novel substituted thiophene derivatives synthesized from 2-amino-4,5,6,7-tetrahydroN-phenylbenzo[b]thiophene-3-carboxamide. Eur. J. Med. Chem. 2010, 45, 5935-5942.

8. Abdel-Rahman, S.A.; El-Gohary, N.S.; El-Bendary, E.R.; El-Ashry, S.M.; Shaaban, M.I. Synthesis, antimicrobial, antiquorum-sensing, antitumor and cytotoxic activities of new series of cyclopenta(hepta)[b]thiophene and fused cyclohepta[b]thiophene analogs, Eur. $J$. Med. Chem. 2017, 140, 200-211.

9. Bertosa, B.; Aleksic, M.; Zamola, G.K.; Tomic, S. QSAR analysis of antitumor active amides and quinolones from thiophene series. Int. J. Pharm. 2010, 394, 106-114.

10. Jose L. Gonzalez, J.L.; Stephens, C.E.; Wenzler, T.; Brun, R.; Tanious, F.A.; Wilson, W.D.; Barszcz, T. Werbovetz, K.A.; Boykin, D.W. Synthesis and antiparasitic evaluation of bis2,5-[4-guanidinophenyl]thiophenes. Eur. J. Med. Chem. 2007, 42, 552-557.

11. Sowmya, D.V.; Teja, G.L.; Padmaja, A.; Prasad, V.K.; Padmavathi, V. Green approach for the synthesis of thiophenyl pyrazoles and isoxazoles by adopting 1,3-dipolar cycloaddition methodology and their antimicrobial activity. Eur. J. Med. Chem. 2018, 143, 891-898.

12. Viswanatha, G.L.; Priyadarshini, B.J.; Krishnadas, N.; Janardhanan, S.; Rangappa, S.; Hanumanthappa, S. Synthesis and antihistaminic activity of 3H-benzo[4,5]thieno[2,3d] [1,2,3] triazin-4-ones. Saudi Pharm. J. 2012, 20, 45-52.

13. Amr A.E.; Sherif, M.H.; Assy, M.G.; Mohamed A. Al-Omar, M.A.; Ragab, I. Antiarrhythmic, serotonin antagonist and antianxiety activities of novel substituted thiophene derivatives synthesized from 2-amino-4,5,6,7-tetrahydroN-phenylbenzo[b]thiophene-3-carboxamide. Eur. J. Med. Chem. 2010, 45, 5935-5942. 
14. Kanojia, R.M.; Salatay, J.J.; Kauman, J. Synthesis and Class III type antiarrhythmic activity of 4-aroyl (and aryl)-1-aralkylpiperazines. Bioorg. Med. Chem. Lett. 2000, 10, 2819-2823.

15. Fabritius, C.H.; Pesonen, U.; Messinger, J.; Horvath, R.; Salo, H.; Zowski, M.G.; Galek, M.; Stefan, K.; Spisak, J.S.; Płachta, M.O.; Buda, A.; Adamczyk, J.; Marcin Król, M.; Prusis, P.; Lupa, M.S.; Mikulski, M.; Kuokkanen, K.; Chapman, H.; Obuchowicz, R.; Korjamo, T.; Jalava, N.; Nowak, M. 1-Sulfonyl-6-piperazinyl-7-azaindoles as potent and pseudoselective 5-HT6 receptor antagonists. Bioorg. Med. Chem. Lett. 2016, 26, 2610-2615.

16. Mohareb, R.M.; Wardakhan W.W.; Ibrahim, R.A. Synthesis of pyridine, pyran and thiazole containing thiophene derivatives and their anti-tumor evaluations. Med. Chem. Res. 2016, $25,2187-2204$.

17. El-Sayed, N.N.E.; Abdelaziz, M.A.; Wardakhan,W.W.; Mohareb, R.M. The Knoevenagel reaction of cyanoacetylhydrazine with pregnenolone: Synthesis of thiophene, thieno[2,3d] pyrimidine, 1,2,4-triazole, pyran and pyridine derivatives with anti-inflammatory and antiulcer activities. Steroids 2016, 117, 98-111.

18. Ibrahim, R.A. Uses of acetoacetanilide for the synthesis of thiophene derivatives with cytotoxic activities. Bull. Chem. Soc. Ethiop. 2017, 31, 519-534.

19. Barnes, D.M.; Haight, A.R.; Hameury, T.; McLaughlin, M.A.; Mei, J.; Tedrowy, J.S.; Toma, J.D.R. New conditions for the synthesis of thiophenes via the Knoevenagel/Gewald reaction sequence. Application to the synthesis of a multitargeted kinase inhibitor. Tetrahedron 2006, 62, 11311-11319.

20. Zita Puterova, Z.; Anita Andicsova, A.; Ve'gh, D. Synthesis of $\pi$-conjugated thiophenes starting from substituted 3-oxopropanenitriles via Gewald reaction. Tetrahedron 2008, 64, 11262-11269. 